\title{
Effects of shear, defocus, and wavefront error on the theoretical performance of the composite infrared spectrometer for Cassini
}

\author{
Anthony J. Martino and John G. Hagopian ${ }^{h}$ \\ 'Laser/Electro-Optics Branch, Code 554, NASA Goddard Space Flight Center, Greenbelt, MD $2(1771$ \\ 'Optics Branch, Code 551, NASA Goddard Space Flight Center, Greenbelt, MD 20771
}

\begin{abstract}
The combined effects on performance of shear between the two arms, defocus of the detector, and difference in wavefront between the two arms of a Fourier transform spectrometer using cube corner retroreflectors were investigated. Performance was characterized by the amplitude of the fringe signals coming from a detector as the path-length difference was scanned. A closed-form expression was found for the combined effects of shear and deficus, and it was found that defocus had no effect in the absence of shear. The effect of wavefront error was incdeled numerically and assumed to be independent of shear and defocus. Results were compared with measurements made on the breadboard and engineering model of the Composite Infrared Spectrometer for the Cassini mission to Saturn, and good agreement was found.
\end{abstract}

Keywords: Cassini, CIRS, Michelson interferometer, Fourier transform spectrometer, reflectance, shear, defocus, wavefront, alignment, modulation

\section{INTRODUCTION}

The Composite Infrared Spectrometer ${ }^{1,2}$ (CIRS) for the Cassini mission to Satum is a Fourier transform infrared spectrometer that contains three Michelson-type interferometers. One is used for spectral measurements in the mid-infrared (MIR) band of 7 to $17 \mu \mathrm{m}$; another is used in the far-infrared (FIR) band of 17 to $1000 \mu \mathrm{m}$. The moving elements of these two interferometers are mounted on a common scan mechanism?. The third is a reference interferometer that measures the position of the scan mechanism.

During the development of the MIR interferometer, it heciune necessiry to detennine the theoretical performance of the real, as opposed to ideal, interferometer. Effects of all known departures from ideal were to be calculated and compared with the measured performance of the actual hardware. Effects that were included in the calculations were reflectances of all surfaces in the beamsplitter/compensator stack, wavefront aberrations due to the figures of these surfaces and the retroreflectors, and two forms of misalignment: shear and defocus. Performance of the interferometer was measured in terms of the observed modulation of the output signal.

\section{CONCEPTS AND DEFINITIONS}

A conceptual sketch of the MIR interferometer is shown in Figure 1. A source is nominally located at une foc:d plane of the input lens. An image is formed, nominally at the focid plante of the output lens. A detector, the area of which defines the field of view, is also located nominally at the sutput focal plante. In the subsequent andilysis, defocus will refer to axial displacement of the source or detector from the input or sutput focal platne.

At the center of the interterometer is a beansplitter/compensaltor pair. The substratte matterial is potatsium bromide (KBr). The beamsplitter coating, which is deposited on the second surtice of the be:unsplitter, is monitlally $50 \%$ reflective and $50 \%$ transmissive for both polarizations across the spectral halldwidth of interest. 


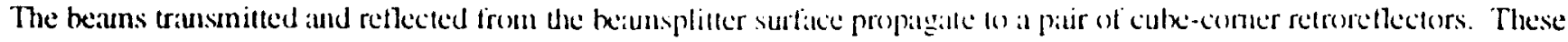
retroreflectors terminate die arms of the Michelson interterometer. The be:ans that return from the retromeflectors are recombined at the beiunsplitter, then fixcused by dhe output kens onto the delector. As the moving retroretlector translites along the optical axis, the path lengul difference he ween the two arms chingess. This gives rise fo al varying signal recorded by the detector. This signal can be characterized by its molulition, given by

$$
M=\frac{S_{\max }-S_{\min }}{S_{\max }+S_{\min }},
$$

where $S$ is the signal recorded by the detector.

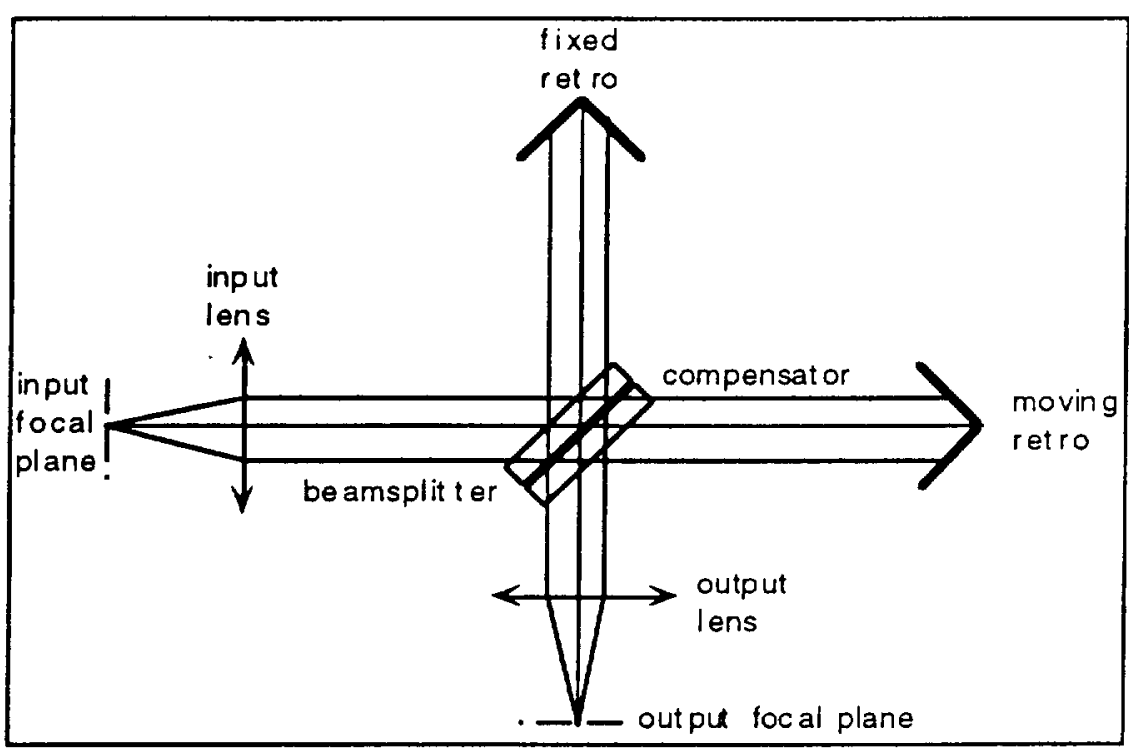

Figure 1. Conceptual sketch of the MIR interferometer.
The actual retlectance of the beamsplitter surfice deviates from ideal performance. Also, there is no antiretlection coating on the first surface of the beansplitter or either surfice of the compensator. The reflectance of uncoated $\mathrm{KBr}$ at the relevant angle of incidence $\left(37^{\circ}\right)$ is high enough that a signiticant amount of light goes into stray hearns that are reflected at surfaces other than the nominal beansplitter surface. These surfaces are slightly tilted with respect to the beamsplitter surface, so the stray beams do not contribute to the modulation of the interferogriun recorded at the detector. However, they do contribute an offset term.

Nominally, the apexes of the cube comers are located on the optical axis, which is detined by the focal point and center of the input lens. In this case, rays in the fixed and moving arms that come from a single raty incident on the beamsplitter meet again when the beams are recombined.

If one or both of the cube coners is displaced laterally from its nominal position, the rays returning from the two arms are displaced. This displacement of the two retuming beams is called shear. Alone, and in combination with defocus, it affects the modulation of the detected signal.

Errors in the surface figures of the beansplitter, compensator, and retroreflectors catuse a viriation of phatie difference across the aperture of the interferometer. This also contributes to a loss of modulation.

Except for shear and defocus, these effects are, to a good approximation. independent of each other. Consequently, they cau be treated separately and then combined to yield a finad result.

\section{SURFACE REFLECTANCE}

It is well known ${ }^{5}$ that, for an ideal Michelson interlenometer, the modulation is given by

$$
M=4 R T \text {. }
$$

where $R$ is the reflectance and $T$ is the transmittance of die be:unsplitter. When there atre additionall reflective surfices

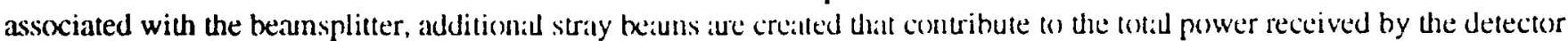
but not to the interferogram.

The zero order beam of the interferoneter consists of mys ulit split and recombinc at the be:unspliter surfaces, and do not reflect at any other beaunsplitter or comperasator surtice. The zero order power that reaches the detector (the main beium) from the moving and fixed arms is given by 


$$
\begin{aligned}
& P_{m m}=P_{i n} T_{u e}{ }^{3} T_{u i}{ }^{2} R_{c i} T_{c i} \\
& P_{m f}=P_{i n} T_{u e}{ }^{4} T_{u i}{ }^{3} R_{c e} T_{c i}
\end{aligned}
$$

where $P_{\text {in }}$ is the input power, the subscripts "c" and " $u$ " meiul "coated" and "uncoilted" with the heamsplitter coating; and "i" and " $\mathrm{e}$ " mean internal and extermal reflection. Then the anplitude of the fringes is

$$
A=2 \sqrt{P_{m f} P_{m m}}=2 \sqrt{T_{u s}{ }^{7} T_{u i}^{5} R_{c e} T_{c i} R_{c i} T_{c i}}
$$

A similar expression can be found for zero order power that is retumed to the source. In the MIR interferometer, the fraction of the input power that reaches the detector in a zero-order bean varies between $11 \%$ and $37 \%$, depending on wavelength and polarization. This leads to modulation of the main beam that varies between $89 \%$ and $97 \%$. The complementary main beam, which returns to the source, accounts for another $32 \%$ i) $78 \%$ of the input power.

Stray beams can be classified as first order, second order, elc., balsed on the number of reflections made at the front beamsplitter surface and the compensator surfaces. Together, these higher-order beams account for $10 \%$ to $35 \%$ of the input power. If we (very roughly) approximate that the higher-order power is split between the detector and the source according to the same ratio as for the zero-order power, and calculate inodulation as

$$
M=\frac{A}{P_{\text {ous }}} \text {, }
$$

where $P_{\text {ow }}$ is the mean power reaching the detector from all beans, we obtain the results shown in Figure 2.

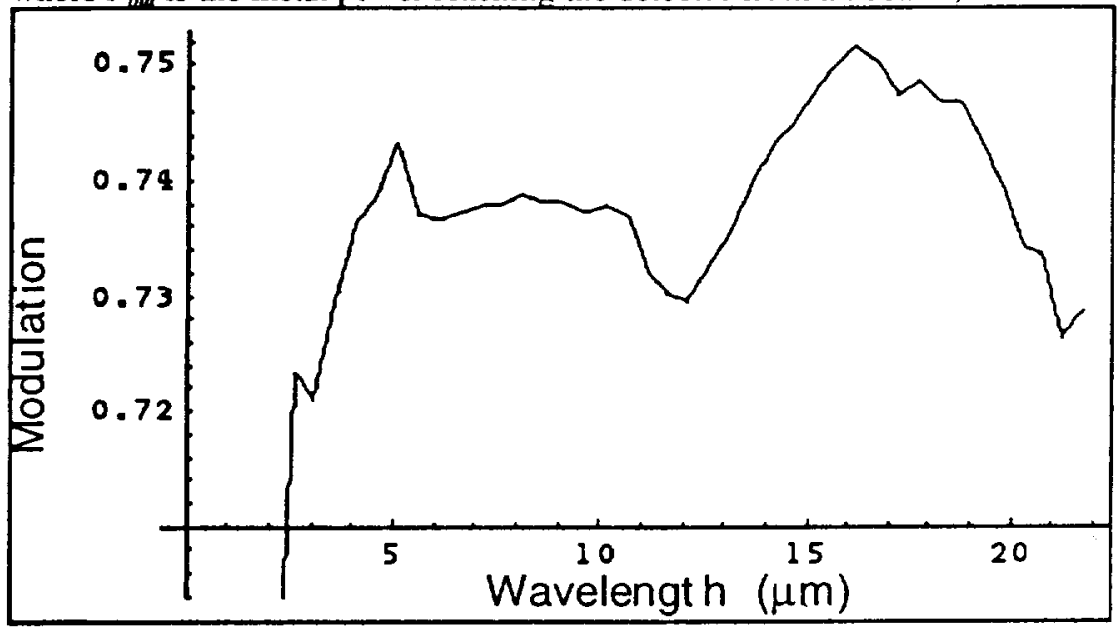

Figure 2. Modulation of the output light due to beansplitter and compensator surface reflectance, averaged between $s$ and $p$ prolarizations.

\section{WAVEFRONT ERROR}

The electric field at a point $(x, y)$ in the aperture, assumning equal powers in the two arms, is

$$
\mathrm{E}(x, y)=\frac{1}{2} \mathbf{E}_{0}\left[e^{i \phi_{1}(x, y)}+e^{i\left(\phi_{1}(x, y)+k z+\Delta \phi_{,}(x, y)\right)}\right]
$$

where $\phi_{1}$ is the phase at $(x, y)$ from one interferometer ium in ridiiuls, $\Delta \phi_{e}$ is the phase difference between the two arms due to wavefront errors, and $z$ is the mean path length dillerence.

The power at the incerference plane is 


$$
\begin{aligned}
P(x, y) & =E(x, y) E^{\circ}(x, y) \\
& =\frac{1}{2} E_{o}{ }^{2}\left[1+\cos \left(k z+\Delta \phi_{e}(x, y)\right)\right] \\
& =P_{0}\left[1+\cos \left(k z+\Delta \phi_{e}(x, y)\right)\right]
\end{aligned}
$$

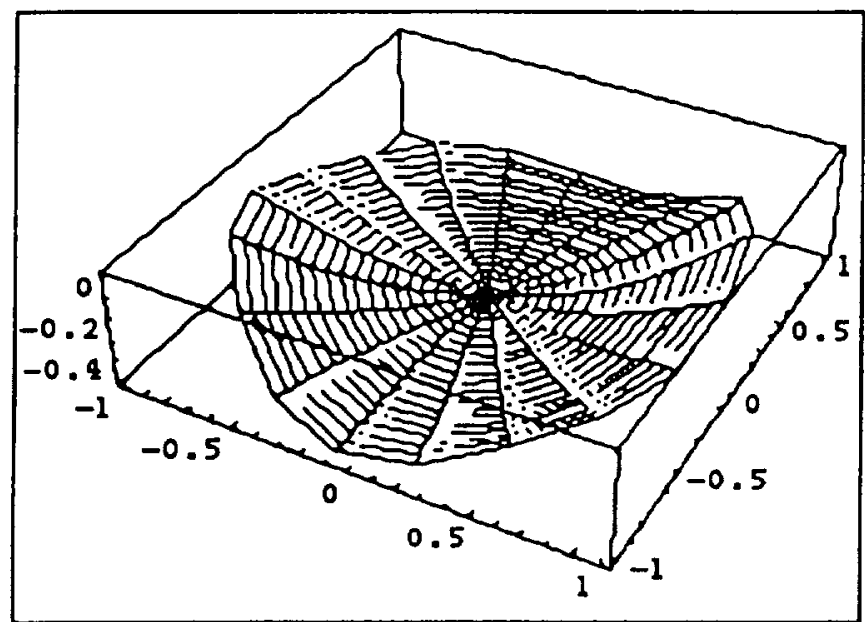

Figure 3. Phase difference in waves (at $9.1 \mu \mathrm{m}$ ) between the fixed and moving arms of the CIRS MIR interferometer due to surface figures. Horizontal units are fractions of pupil radius.

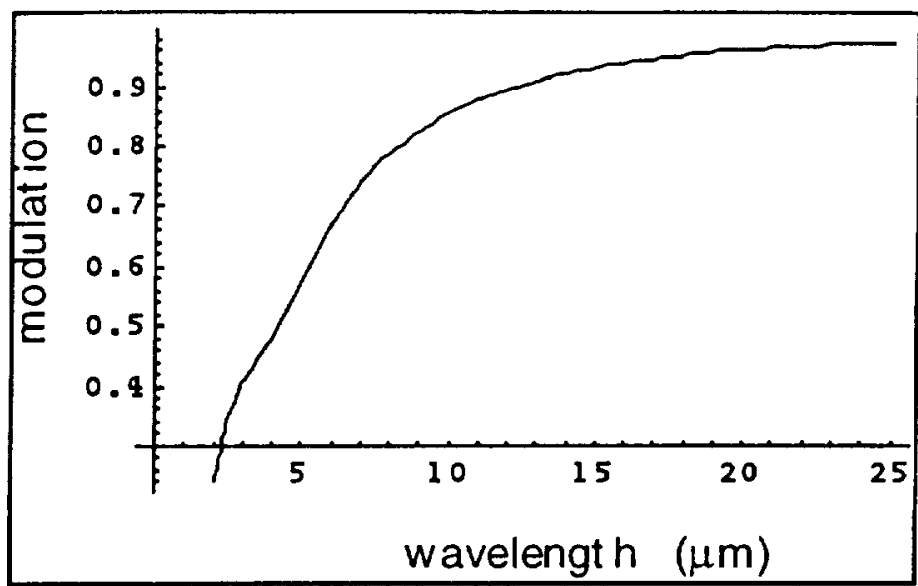

Figure 4. Modulation of the CIRS MIR interferometer due to the wavefront difference shown in Figure 3.

The interferogram is produced by focusing the total power in the aperture to a spot, then capturing the spot on a detectir:

$$
\begin{aligned}
I(z) & =\int_{A} P_{0}\left[1+\cos \left(k z+\Delta \phi_{e}(x, y)\right)\right] d x d y \\
& =P_{0} A+P_{0} \int_{A} \cos \left(k z+\Delta \phi_{e}(x, y)\right) d x d y \\
& =P_{0} A+P_{0} \cos k z \int_{A} \cos \Delta \phi_{e}(x, y) d x d y-P_{0} \sin k z \int_{A} \sin \Delta \phi_{e}(x, y) d x d y
\end{aligned}
$$

Let

$$
\begin{aligned}
& H_{1}=\int_{A} \cos \Delta \phi_{e}(x, y) d x d y \\
& H_{2}=\int_{A} \sin \Delta \phi_{e}(x, y) d x d y
\end{aligned}
$$

Then

$$
\begin{aligned}
I(z) & =A P_{0}+P_{0} H_{1} \cos k z-P_{0} H_{2} \sin k z \\
& =A P_{0}+P_{0} \sqrt{H_{1}{ }^{2}+H_{2}{ }^{2}}(\cos (k z+\Phi))
\end{aligned}
$$

where $\tan \Phi=\frac{H_{2}}{\Pi_{1}}$. Then the me:un of the interterogran is $A P_{0}$ and the inodulation is 


$$
M=\frac{\sqrt{H_{1}^{2}+H_{2}^{2}}}{A}
$$

For some wavefront shapes, $H_{1}$ and $H_{2}$ could be calculated antlytically. In practice, it is necessatry to compute them numerically from measured data. To inodel the CIRS MIR interferometer, surfice figures or wavefront distontions were measured for each component using a Zygo interterometer. Wavefronts trom the lixed and moving arms, and wavefront difference, were computed using these measured datti. Figure 3 is a plot of computed wavefront difference at $7.1 \mu \mathrm{m}$ wavelength, and Figure 4 is a plot of the resulting modulation as a function of wavenumber.

\section{SHEAR AND DEFOCUS}

A treatment of the effect of shear in a cube-comer Michelson interferometer has been published by Murty ${ }^{6}$. For a cube-comer Michelson interferometer with a circular field of view, no defocus, at zero path difference, the modulation is given by

$$
M=\frac{J_{1}(2 \sigma \sqrt{\pi \Omega})}{\sigma \sqrt{\pi \Omega}}
$$

where $\sigma$ is the wavenumber, $s$ is the wavefront shear (twice the cube corner displacement), and $\Omega$ is the solid angle subtended by the field of view. Kauppinen and Satarinen ${ }^{7}$ have extended this theory to predict line shape distortions in the spectral domain.

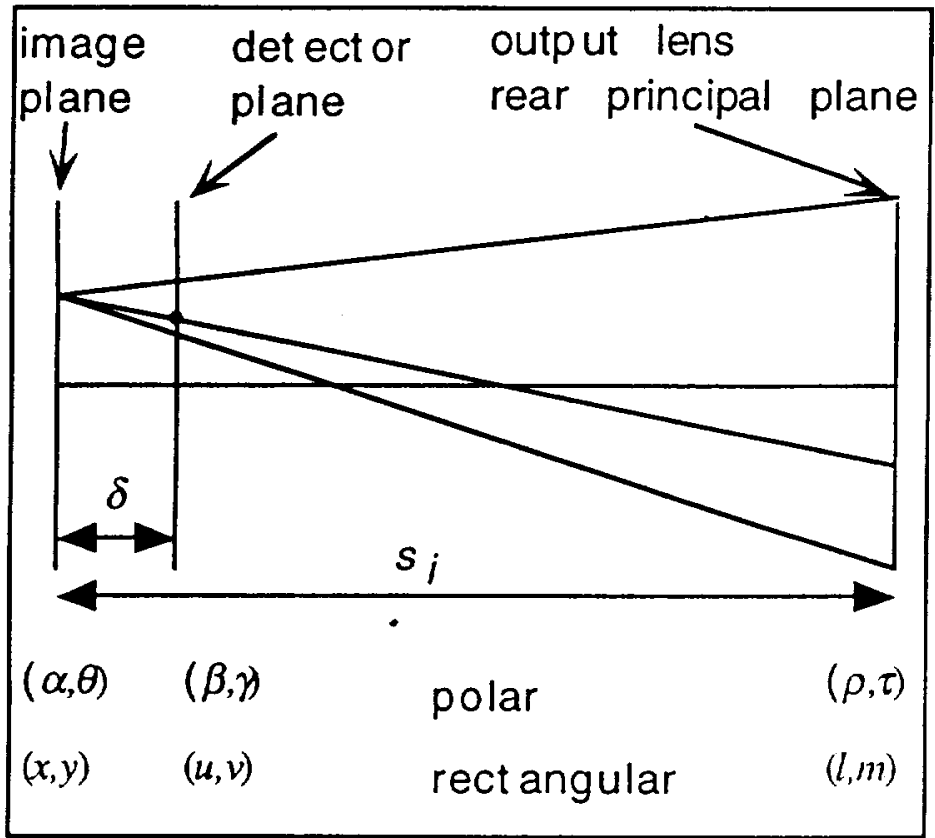

Figure 5. Geometry for defocus/shear theory, showing significant planes and polar and rectiungular coordinatess used therein.
Satrinen and Kauppinen ${ }^{8}$ have examined the effect of a defocused point source on the interferogram and in the spectral domain, expanding on work by Guelachvili?' Kunz and Goorvitch ${ }^{10}$ have exannined the combined effects of source defocus and mirror misalignment in a Michelson interferometer that uses plane mirrors. Here we present a treatunent of the combined effects of defocus and shear on the modulation at ZPD of a cube-comer Michelson interferometer looking at an object that fills a small but nonzero tield of view.

The geometry is shown in Figure 5. Each point in the source (not shown) is imaged to a point in the image pline (nominally the focal plane of the output lens) at a distance $S_{i}$ trom the rear principal plane of the output lens. A planar detector is located at some distance $\delta$ from the unage plane. For each point $(u, v)$ in the detector plane and $(l, m)$ in the pupil, there is a corresponding point $(x, y)$ in the image plane.

The pupil of the optical system is circular, with radius $R_{p}$. In gencral, the source is not at the focid plane of the input lens, so the wavefront incident on the output lens is spherical with radius $R_{w}$. In this intalysis, $R_{w}$ is assumed

to be much greater than the maximum path length difference between the (wo) ams of the interferometer, so the wavefronts from the two arms can be treated as having the siane radius of curvalture.

Consider a point on the $y$-axis in the image plante. The watrelfont dhat is focused onto it is descrithed partaxially in the pupil plane as 
$z(l, m)=m \frac{y}{s_{i}}+\frac{l^{2}+m^{2} /\left(1-\left(y / s_{i}\right)^{2}\right)}{2 R_{w}}$

A wavefront that is sheared widh lespect 10 this onc by $s$ is

$z(l, m)=(m+s) \frac{y}{s_{i}}+\frac{l^{2}+(m+s)^{2} /\left(1-\left(y / s_{i}\right)^{2}\right)}{2 R_{w}}$

Then the phase difference between the two waves is

$\Delta \Phi(0, y, l, m)=\frac{2 \pi}{\lambda}\left(s \frac{y}{s_{i}}+\frac{2 s m+s^{2}}{2 R_{w}\left(1-\left(y / s_{i}\right)^{2}\right)}\right)$

Since the image height is much smaller than the inage distance, we can eliminate terms of order 2 or higher in $y / s_{\text {, }}$ yielding

$\Delta \Phi(0, y, l, m)=\frac{2 \pi}{\lambda}\left(s \frac{y}{s_{i}}+\frac{2 s m+s^{2}}{2 R_{w}}\right)$.

It can be shown that for arbitrary tield points, under the siune conditions, the same result holds. Then

$P(x, y, l, m, z)=P_{0}+P_{0} \cos k\left(z+\frac{y s}{s_{i}}+\frac{s^{2}}{2 R_{w}}+\frac{s m}{R_{w}}\right)$

The power that strikes a point $(u, v)$ on the detector is

$P(u, v, z)=\int_{0}^{\infty} \rho d \rho \int_{0}^{2 \pi} d \tau P(\alpha, \theta, \rho, \tau)$

where $(u, v),(\alpha, \theta)$, and $(\rho, \tau)$ are all collinear. Substituting (17) into (18) and using some geometry to express image plane coordinates in terms of pupil and detector plane coordinates, we obtain

$P(u, v, z)=\pi R_{p}^{2} P_{0}+P_{0} \int_{0}^{\infty} \rho d \rho \int_{0}^{2 \pi} d \tau \cos k\left[\begin{array}{l}z+\left(\frac{s}{R_{w}}-\frac{s \delta}{s_{i}\left(s_{i}-\delta\right)}\right) \rho \sin \tau \\ +\frac{s \delta v}{s_{i}\left(s_{i}-\delta\right)}+\frac{s v}{s_{i}}+\frac{s^{2}}{2 R_{w}}\end{array}\right]$,

which evaluates to

$$
\begin{aligned}
& P(u, v, z)=\pi R_{p}^{2} P_{0} \\
& +\frac{2 \pi P_{0} R_{p}}{k\left[\frac{s}{R_{w}}-\frac{s \delta}{s_{i}\left(s_{i}-\delta\right)}\right]} J_{1}\left(k R_{p}\left[\frac{s}{R_{w}}-\frac{s \delta}{s_{i}\left(s_{i}-\delta\right)}\right]\right) \cos \left(k\left[\frac{s v}{s_{i}-\delta}+\frac{s^{2}}{2 R_{w}}+z\right]\right) .
\end{aligned}
$$

In the case of a rectangular detector with dimensions $\Delta u$ and $\Delta v$, the power integrated across the detector is 


$$
\begin{aligned}
& P(z)=\pi R_{p}{ }^{2} P_{0} \Delta u \Delta v \\
& +2 \pi P_{0} R_{p}^{2} \Delta u \frac{J_{1}\left(\Psi_{1}\right)}{\Psi_{1}} \frac{s_{i}-\delta}{k s}\left[\begin{array}{l}
\left(\sin \frac{k s v_{\text {niux }}}{s_{i}-\delta}-\sin \frac{k s v_{\min }}{s_{i}-\delta}\right) \cos k\left[\frac{s^{2}}{2 R_{w}}+z\right] \\
+\left(\cos \frac{k s v_{\text {mixx }}}{s_{i}-\delta}-\cos \frac{k s v_{\text {min }}}{s_{i}-\delta}\right) \sin k\left[\frac{s^{2}}{2 R_{w}}+z\right]
\end{array}\right],
\end{aligned}
$$

where

$$
\Psi_{1}=k R_{p}\left[\frac{s}{R_{w}}-\frac{s \delta}{s_{i}\left(s_{i}-\delta\right)}\right] \text {. }
$$

Then the modulation is

$$
M(s, \delta, \Delta v)=2 \frac{J_{1}\left(\Psi_{1}\right)}{\Psi_{1}} \frac{\sin \left(\Psi_{2}\right)}{\Psi_{2}}
$$

where

$$
\Psi_{2}=\frac{k s \Delta v}{2\left(s_{i}-\delta\right)} \text {. }
$$

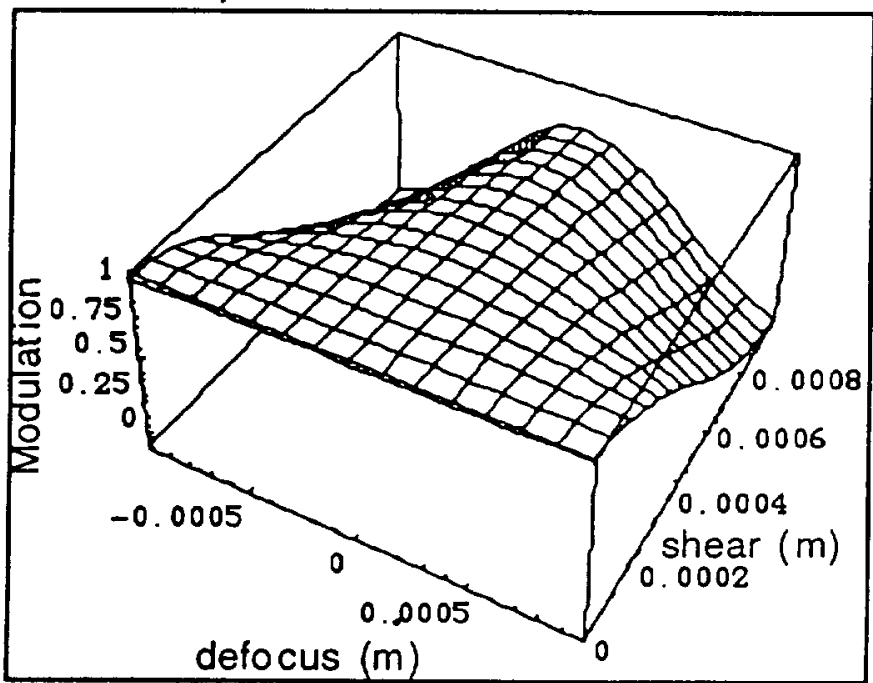

Figure 6. Effect of shear and defocus on modulation of the CIRS MIR interferometer at $10 \mu \mathrm{m}$ wavelength.
An interesting feature of this result is that, while shear affects the modulation by itself, defocus at the output affects Inodulation only in the presence of shear. With shear present, modulation is maximized by placing the detector not at the focal plane of the output lens, but at the image of the source. (The image of the source coincides with the focal plane of the output lens if there is no defocus at the input.)

Figure 6 illustrates the effect of shear and output defocus on the modulation of the CIRS MIR interferometer. The detector in this instunce is an array of pixels, each of which can be regarded is a $200 \mu \mathrm{m}$ square.

\section{COMPARISON OF THE COMBINED EFFECTS WITH EXPERIMIENT}

All of these effects were combined to produce predictions of the performance of the CIRS MIR interferometer. The predictions were compared with measurements made on the engineering and tlight moxdels of the instrument. The details of the experimental measurements are described in ansuler paper".

In Figure 7, we show predictions of measured spectra using a 50)" (C blackbody source under various conditions. The calculations include the effects described ibove and the relitive specurd responsivily of a HoCdTe detector. Figure 8 is an example of actual data ineasured under comesponding conditions in a CIRS bre:idbo:ird, using a detector simnilar but not identical to the one whose responsivity was used in the calculation. 


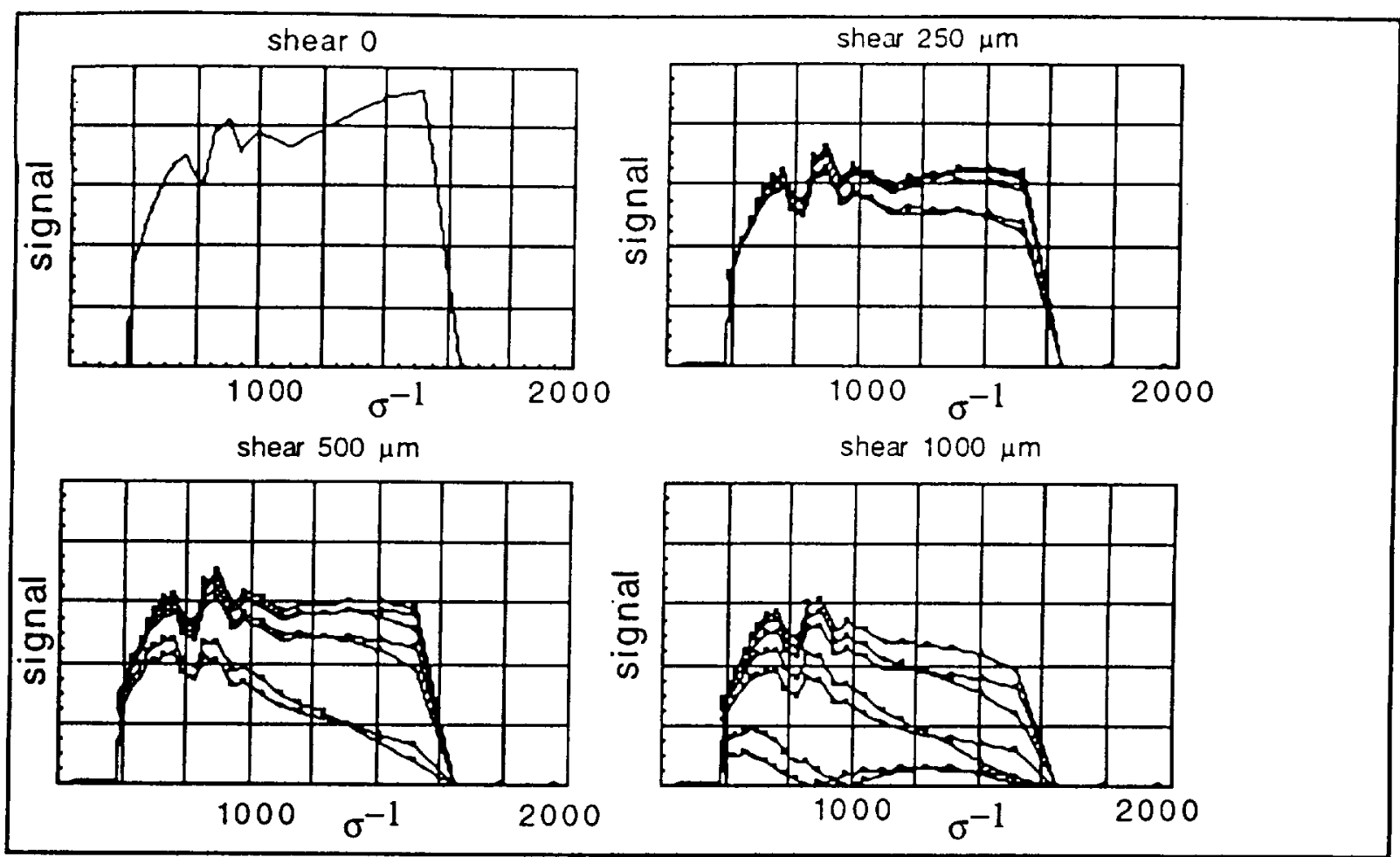

Figure 7. Predicted performance of the CIRS MIR interferometer ineasuring a $773 \mathrm{~K}$ blackbody spectrum. Effects of beamsplitter/compensator reflectance, surface tigures, shear, output defocus, and detector responsivity are included. Each plot shows detector response in abbitrary units as a function of wavenumber with defocus (from top) $0, \pm 250 \mu \mathrm{m}, \pm 500 \mu \mathrm{m}$, and $\pm 1000 \mu \mathrm{m}$.

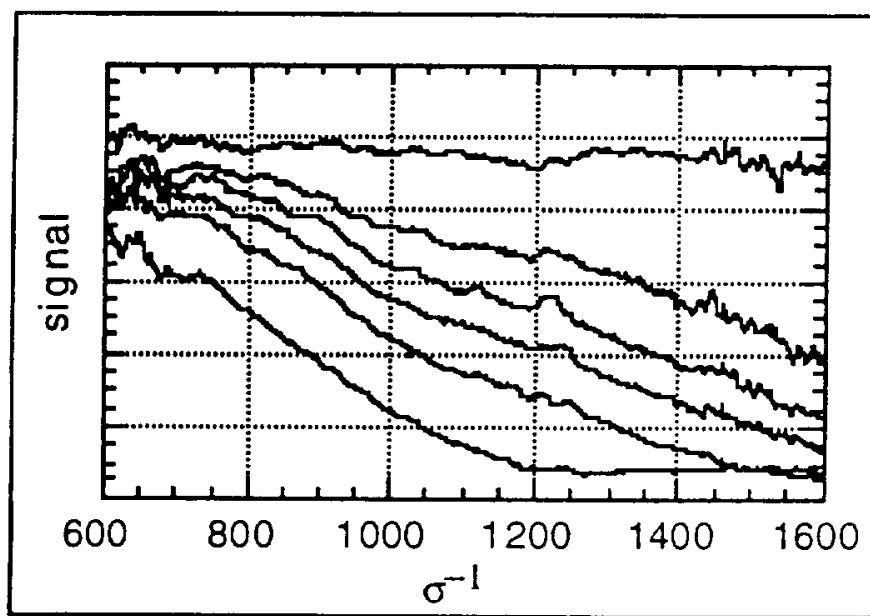

Figure 8. Spectrum of a $773 \mathrm{~K}$ blackbody, meisured will (from top) $200 \mu \mathrm{m}$ shear and no defocus, $10(x) \mu \mathrm{m}$ of sheitr and $0,200 \mu \mathrm{m}, 300 \mu \mathrm{m}, 400 \mu \mathrm{m}$, and $600 \mu \mathrm{m}$ of output defocus.
With no she:tr, the theoretical plot is simply the blackbody spectrum multiplied by the relative responsivity of the detector. In the absence of defocus, the effect of shear is noticeable but mild, being most pronounced at higher wavenumbers (shorter wavelengths).

As shear is increased, the effects of defocus become more and more pronounced. Large degradations appear first at higher wavenumbers, then propagate to lower wavenumbers as the she:ur or defocus increases. Note that there is little difference hetween the elfects of positive and negative defocus.

The experimentill results agree well with the theory. As expectid, $200 \mu \mathrm{m}$ of shear with no defocus shows a ne:rly flat spectrum. With $1(k)() \mu \mathrm{m}$ of shear, the performance at higher wavenumbers is degraded markedly even with no defocus. Perfominne at the lower wavenumbers is still affected only slighly. As delicus increases, pertionnance drops quickly at high wavenumbers and more slowly at low wavenumbers. At the largest defocus $(1000 \mu \mathrm{m})$, the pertormance actually recovers slightly at the highest watvenumbers.

The theory presented in this paper was used in the diagnesis and chataterization of UL CIRS instrument. Widh this

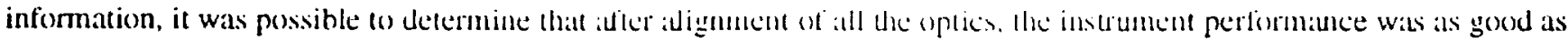
could be obtained with the fiabricalted hardiwite. 


\section{REFERENCES}

1. V. Kunde et. al., "Cassini infrared Fourier spectroscopic investigation," Proc. SPIE 2803, pp. 162-177, 1996.

2. P. W. Maymon et al., "Optical design of the composite infrured spectometer (CIRS) for the Cassini mission," Proc. SPIE 1945, pp. 100-111, 1993.

3. C. F. Hakun and K. A. Blumenstock, "A cryogenic scan mechamism tor use in Fourier trinsform spectrometers," in NASA Johnson Space Flight Center 29th Aerospace Mechanisms. Sympositum, np. 316-333, 1995.

4. A. J. Martino and D. M. Cornwell, "Reference interferometer using a semiconductor laser/LED reference source in a cryogenic Fourier-transform spectrometer," Proc: SPIE 3435, in press, 1998.

5. R.J. Bell, Introductory Fourier Transform Spectroscopy, p. 112, Academic Press, New York, 1972.

6. M. V. R. K. Murty, "Some more aspects of the Michelson interferometer with cube comers," J. Opt. Soc. Am. 50, pp. 7-10, 1960.

7. J. Kauppinen and P. Saarinen, "Line-shape distortions in misaligned cube comer interferometers," Appl. Opt. 31, pp. 6974, 1992.

8. P. Saarinen and J. Kauppinen, "Spectral line-shape distortions in Michelson interferometers due to off-focus radiation source," Appl. Opt. 31, pp. 2353-2359, 1992.

9. G. Guelachvili, "Distortions in Fourier spectra and diagnosis," Spectrometric Techniques, G. Vanasse, V. II, pp. 1-62, Academic Press, New York, 1981.

10. L. W. Kunz and D. Goorvitch, "Combined effects of a converging beiun of light and mirror misalignment in Michelson interferometry," Appl. Opt. 13, pp. 1077-1079, 1974.

11. J. G. Hagopian et. al, "Shear/defocus sensitivity of the mid-infrared channel (MIR) of the connosite infrared spectrometer (CIRS) for the Cassini mission to Satum," Proc. SPIE 3435, in press, 1998. 